\title{
EL ESTUDIO DE LOS EFECTOS DE LA PRESENCIA DE BURBUJAS DE HELIO EN LA HIDRODINÁMICA DE LOS CANALES DE HCLL
}

\author{
D. M. L. de PAULA ${ }^{1}$, E. MAS DE LES VALLS ${ }^{2}$, A. M. O. SIQUEIRA ${ }^{1}$ e L. BATET ${ }^{3}$ \\ ${ }^{1}$ Universidade Federal de Viçosa, Departamento de Química e Engenharia Química \\ ${ }^{2}$ Universitat Politècnica de Catalunya, Departamento de Máquinas e Motores Térmicos \\ ${ }^{3}$ Universitat Politècnica de Catalunya, Departamento de Física e Engenharia Nuclear \\ E-mail para contato: dayanamlouzada@gmail.com
}

\begin{abstract}
RESUMO - En este trabajo se han estudiado los efectos de la presencia de burbujas de helio en la hidrodinámica de los canales de HCLL. Por medio del código OpenFOAM, se há desarrollado y validado la metodología de fluido bifásico a ser utilizada. Los resultados han mostrado que de hecho la nucleación heterogénea de burbujas puede tener una influencia importante en el comportamiento hidrodinámico del metal líquido, lo que puede afectar negativamente a la eficiencia de generación y de extracción de tritio. Las conclusiones más importantes acerca del análisis del breeding blanket fueron: (1) las micro burbujas afectan mucho más el comportamiento del flujo que las nano burbujas; (2) las fuerzas de drag, lift y virtual mass tienen gran influencia en el flujo bifásico; (3) la magnitud de la generación de helio afecta poco a la hidrodinámica; y (4) para las nano burbujas, cuanto más elevado es el Reynolds, menor es la influencia en la hidrodinámica del metal líquido.
\end{abstract}

\section{INTRODUCCIÓN}

El uso de la fusión nuclear como una fuente de energía alternativa es una propuesta muy atractiva, pues se trata de una tecnología limpia, segura y muy productiva. Por otro lado, un gran desafío de esta tecnología es la obtención del tritio, uno de los combustibles necesarios para la reacción de fusión. Como esto no está disponible en la naturaleza, el tritio es producido en los breending blankets, por medio de la interacción de los neutrones que escapan del plasma con el litio contenido en el blanket como descripto por la Ecuación 1 e la Ecuación 2.

$$
\begin{aligned}
& { }^{6} \mathrm{Li}+\mathrm{n} \rightarrow{ }^{3} \mathrm{H}+4 \mathrm{He}+4,78 \mathrm{MeV} \\
& { }^{7} \mathrm{Li}+\mathrm{n} \rightarrow{ }^{3} \mathrm{H}+{ }^{4} \mathrm{He}+\mathrm{n}-2,47 \mathrm{MeV}
\end{aligned}
$$

Un trabajo experimental, conocido como LIBRETTO (Conrad et al., 1991), estudió la liberación de tritio de la aleación eutéctica $\mathrm{Pb}-15,7 \mathrm{Li}$. Durante este experimento, se detectó la formación de burbujas de helio en el circuito de HCLL y en los sistemas auxiliares. El helio es insoluble en metal líquido, así como otros gases nobles y esta característica, juntamente con las grandes tasas de producción de este gas en los sistemas de HCLL, lleva a elevadas concentraciones 
de este componente por todos los canales de metal líquido, lo que puede provocar el fenómeno de nucleación de helio como verificado en el estudio experimental (Batet et al., 2011).

Como la nucleación de burbujas de helio puede afectar el funcionamiento de los breeding blankets, estudiar su grado de influencia en la eficiencia de estos sistemas es de gran relevancia para la tecnología de fusión nuclear. Luego, este proyecto se ha centrado en el estudio de la influencia de diferentes tamaños de burbuja, factores de generación de helio y Reynolds del metal en la hidrodinámica de los canales de HCLL. El estudio numérico se ha desarrollado con el solver twoPhaseEulerFoam de la herramienta de código libre OpenFOAM.

\section{DESCRIPCIÓN GENERAL DEL MODELO}

Para la simulación numérica, el solver twoPhaseEulerFoam (ya validado en un trabajo anterior) ha sido elegido, pues soluciona problemas de fluido dinámica en sistemas bifásicos de dos fluidos incompresibles, donde una de las fases está dispersa. Este modelo se encuentra basado en el enfoque Euler-Euler, tiene en cuenta las contribuciones de las fuerzas de lift, drag y virtual mass.

Las ecuaciones básicas de este modelo son las ecuaciones de Navier-Stokes representadas por la ecuación de continuidad para cada fase $\varphi$ (Ecuación 3) y por la ecuación de conservación del momento (Ecuación 4):

$$
\begin{aligned}
& \frac{\partial}{\partial \mathrm{t}}\left(\alpha_{\varphi} \rho_{\varphi}\right)+\nabla \cdot\left(\alpha_{\varphi} \rho_{\varphi} \mathrm{U}_{\varphi}\right)=0 \\
& \frac{\partial}{\partial \mathrm{t}}\left(\alpha_{\varphi} \rho_{\varphi} \mathrm{U}_{\varphi}\right)+\nabla \cdot\left(\alpha_{\varphi} \rho_{\varphi} \mathrm{U}_{\varphi} \mathrm{U}_{\varphi}\right)+\nabla \cdot\left(\alpha_{\varphi} \mathrm{Re}_{\varphi, \text { eff }}\right)=-\alpha_{\varphi} \nabla \mathrm{p}+\alpha_{\varphi} \rho_{\varphi} \mathrm{g}+\mathrm{M}_{\varphi}
\end{aligned}
$$

Por otro lado, para la solución del caso en estudio, se ha añadido un término fuente $\left(\mathrm{S}_{\alpha}\right)$ a la ecuación de continuidad en el twoPhaseEulerFoam. Este término se refiere a la nucleación heterogénea del helio definida para todas las paredes del canal HCLL. El término $S_{\alpha}$ fue estimado para una determinada distancia de la pared del canal. Fuera de esta región $S_{\alpha}$ es igual a cero.

$$
\frac{\partial}{\partial \mathrm{t}}\left(\alpha_{\varphi} \rho_{\varphi}\right)+\nabla \cdot\left(\alpha_{\varphi} \rho_{\varphi} \mathrm{U}_{\varphi}\right)=\mathrm{S}_{\alpha}
$$

\section{CASO DE ESTUDIO}

A fin de caracterizar la naturaleza del flujo bifásico del HCLL, la geometría utilizada fue un canal simplificado en 2D. Este canal estudiado corresponde al canal de entrada y salida de litioplomo, entre dos placas refrigerantes y placas de refuerzo, donde el metal líquido entra por el canal inferior con una velocidad $\mathrm{U}_{0}$. El canal incluye el hueco que une los canales de entrada y salida en la primera pared y, por esto, se denomina canal de curva en $\mathrm{U}$. 


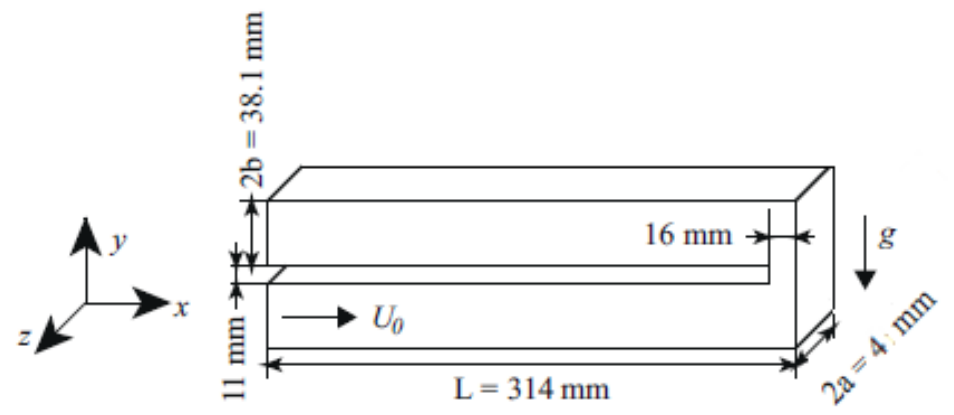

Ilustración 1- Geometría 2D del canal de metal líquido.

Además, las propiedades de los fluidos han sido determinadas a una temperatura de $450^{\circ} \mathrm{C}$. Para la mezcla de metal liquido litio-plomo (Pb-15,7Li) los datos han sido determinados por Mas de les Valls et al. (2008) y los datos del gas helio han sido obtenidos a partir de Perry et al. (2008).

Las simulaciones se han realizado con tres mallas diferentes, una malla gruesa - M1 (3092 volúmenes), una malla fina - M2 (7706 volúmenes) y una malla doble fina - M3 (61648 volúmenes), como se puede observar en la Ilustración 2. M2 y M3 son más complejas y para los cálculos han requerido la función adjustTimeStep para mejor control del número de Courant. En contrapartida, la malla M1, que es una malla más simplificada que las anteriores, ha solucionado los testes con un buen número de Courant y con un timeStep de magnitud fijada en 10-5s, más rápido de lo que se ha utilizado para M2 y M3.

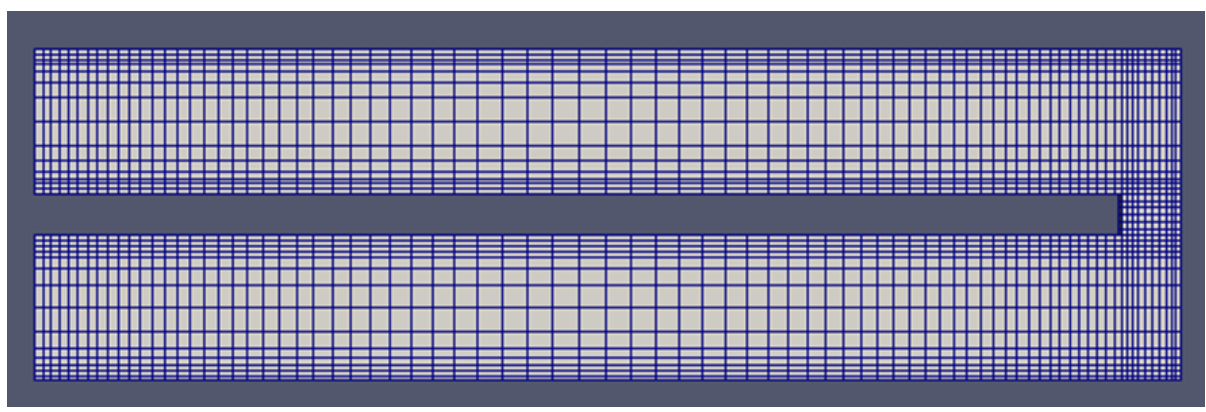

(a)

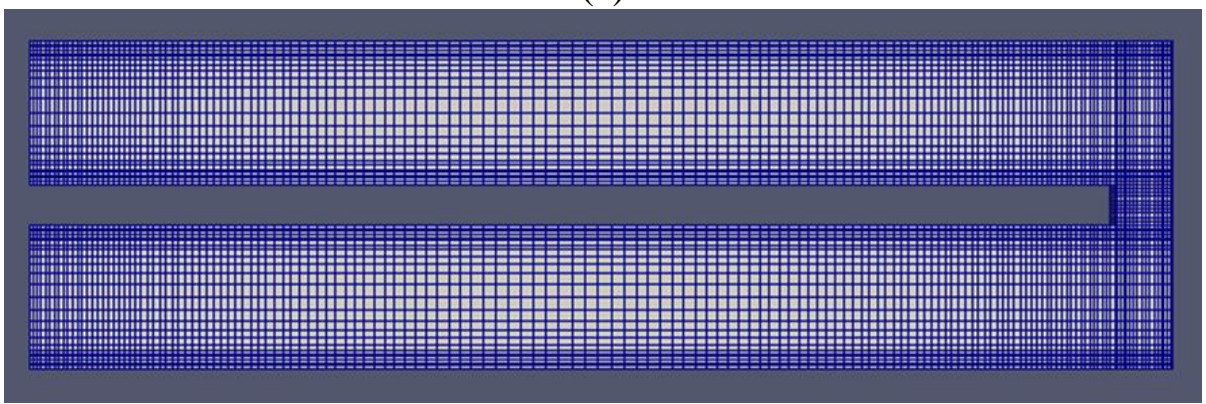

(b) 


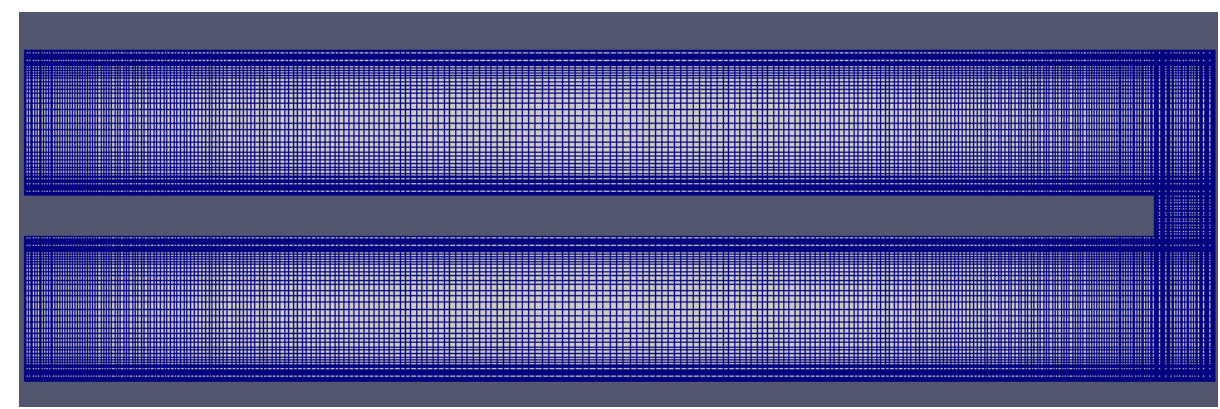

(c)

Ilustración 2 - (a) M1, (b) M2 y (c) M3.

\section{RESULTADOS Y DISCUSIÓN}

Como aún no se conoce el diámetro de las burbujas que se forman dentro de los canales de metal líquido, ni se sabe exactamente la tasa de generación de helio, las soluciones del presente trabajo se han basado en el estudio del efecto general de estos factores en la hidrodinámica del metal líquido $\mathrm{y}$, por lo tanto, diferentes valores de estos parámetros han sido testados durante las simulaciones (Tabla 1). Además, de la misma manera, para analizar la influencia del Reynolds del metal liquido en el comportamiento del flujo bifásico, dos valores de velocidad inicial del metal líquido han sido probados, como también se puede observar en la Tabla 1.

Tabla 1 - Parámetros y respectivos valores testados.

\begin{tabular}{cc}
\hline Parámetros & Valores \\
\hline Diámetro de burbuja de helio & $\mathrm{d}=10^{-6}$ (Micro) \\
& $\mathrm{d}=10^{-6}$ (Nano) \\
Factor de generación de burbujas & $\mathrm{S}_{\alpha}=10^{-9}$ \\
& $\mathrm{~S}_{\alpha}=10^{-5}$ \\
& $\mathrm{~S}_{\alpha}=10^{-4}$ \\
Reynolds de la fase continua & $\mathrm{S}_{\alpha}=2.10^{-4}$ \\
& $\mathrm{Re}=10\left(\mathrm{Ub}=3,14.10^{-5}\right)$ \\
& $\mathrm{Re}=500\left(\mathrm{Ub}=1,71.10^{-3}\right)$ \\
\hline
\end{tabular}

En el primer momento, las simulaciones han sido realizadas para el diámetro de tamaño micrométrico con las mallas M1, M2 y M3. Los resultados han mostrado una buena reproducibilidad entre las tres mallas. En la Ilustración 3, Ilustración 4 e Ilustración 5 es posible analizar el obtenido para M3.

A partir de estas imágenes, se puede afirmar que el tamaño micro de las burbujas ha afectado la hidrodinámica del flujo, puesto que la velocidad que el metal líquido ha alcanzado dentro del canal es mucho superior a su velocidad inicial. Además, debido a la fuerza de empujo, las burbujas generadas se han acumulado en las paredes superiores de ambos los canales y debido a la fuerza de arrastre, las burbujas han sido arrastradas por el flujo hasta la curva estrecha, donde han salido a una velocidad más elevada juntamente con el chorro de metal líquido que se ha formado en esta región. 
A respecto de los otros parámetros en estudio, es posible decir también que con el microdiámetro, el Reynolds de flujos laminares prácticamente no ha provocado cambios significativos en la hidrodinámica puesto que la velocidad de entrada del metal líquido ha influido poco en el perfil de velocidad en el canal.

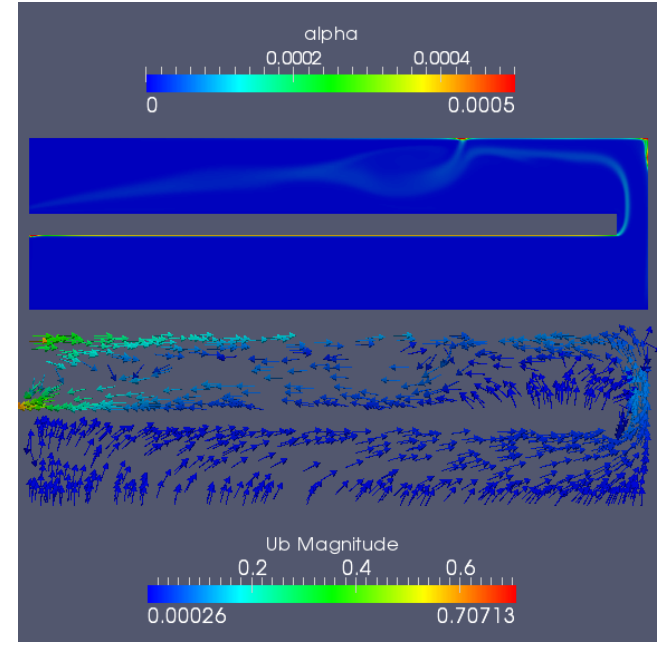

(a) $\operatorname{Re}=10$

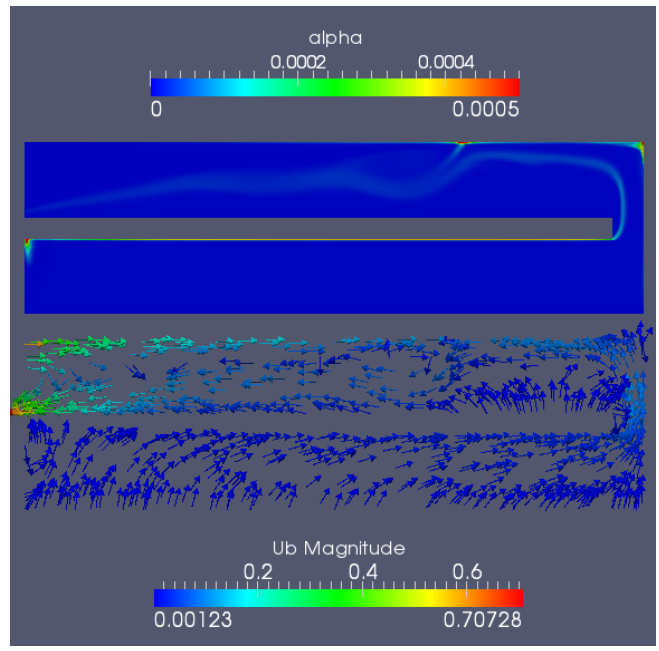

(b) $\operatorname{Re}=500$

Ilustración 3 - Comportamiento de la fracción de volumen de la fase dispersa y el perfil de la velocidad de la fase continua para $\mathrm{S}_{\alpha}=10^{-5}$ (M3).

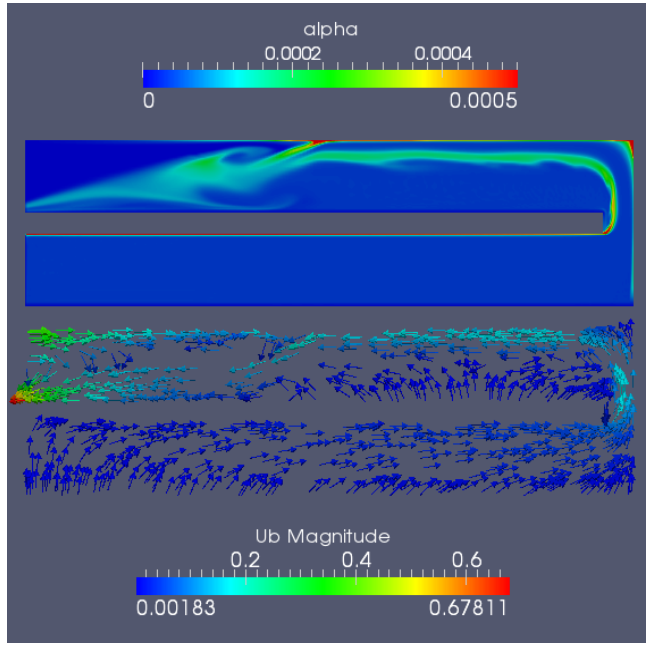

(a) $\operatorname{Re}=10$

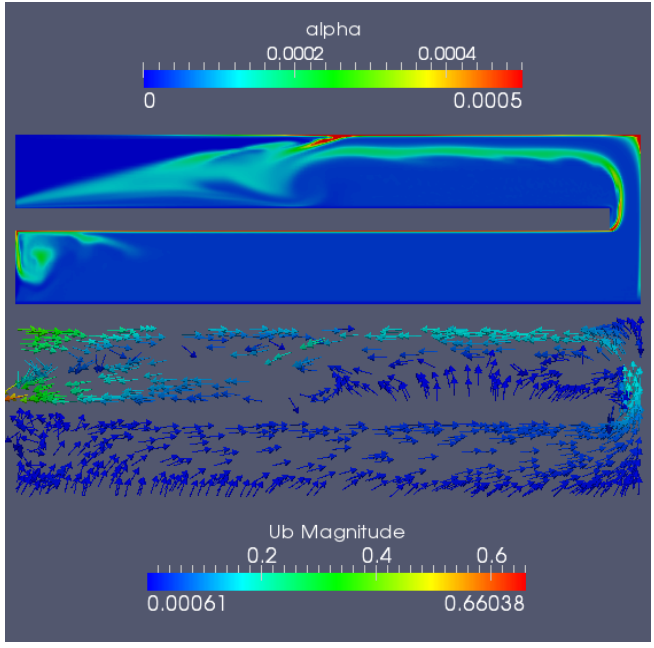

(b) $\operatorname{Re}=500$

Ilustración 4 - Comportamiento de la fracción de volumen de la fase dispersa y el perfil de la velocidad de la fase continua para $\mathrm{S}_{\alpha}=10^{-4}$ (M3). 


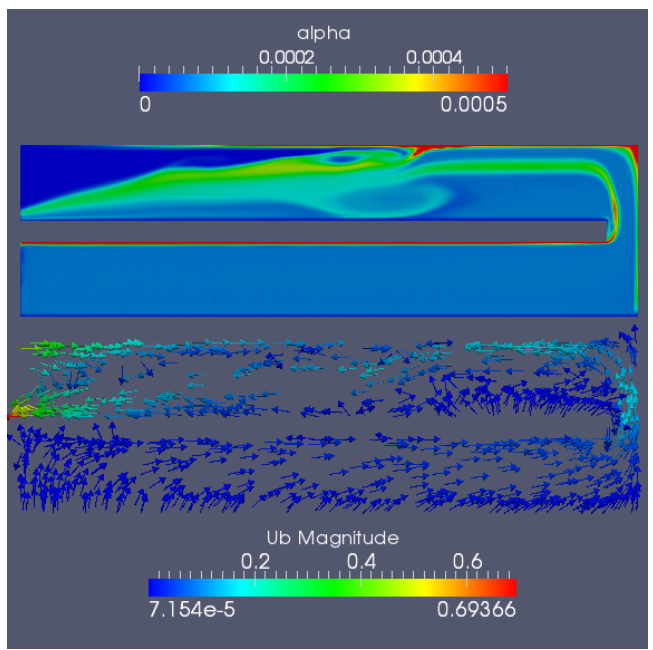

(a) $\operatorname{Re}=10$

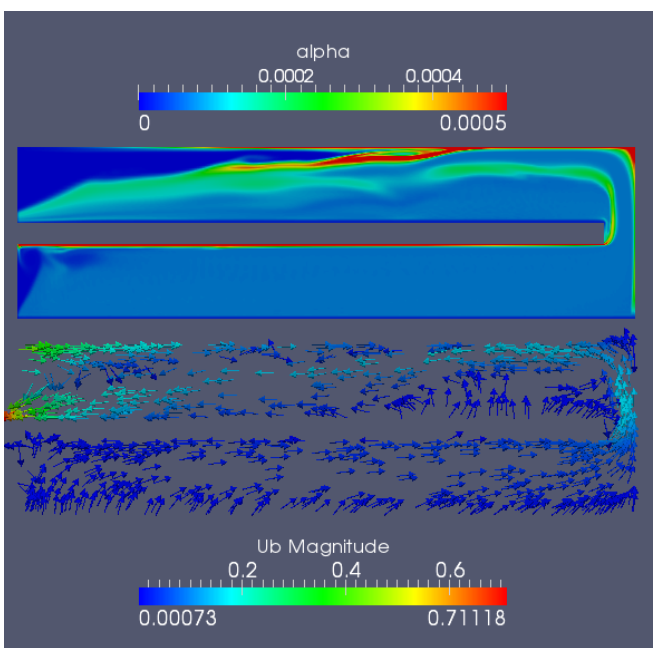

(b) $\operatorname{Re}=500$

Ilustración 5 - Comportamiento de la fracción de volumen de la fase dispersa y el perfil de la velocidad de la fase continua para $\mathrm{S}_{\alpha}=2 \cdot 10^{-4}(\mathrm{M} 3)$.

Los diferentes valores de generación también han afectado poco el comportamiento final del fluido. Comparando los resultados para los factores de generación de burbujas de helio (Ilustración 3, Ilustración 4 e Ilustración 5) se ha observado que la diferencia principal se ha radicado básicamente en el comportamiento del flujo en el canal de salida y en la concentración de burbujas a lo largo del canal U, pues, evidentemente, cuanto menor es el valor de generación de helio, menos burbujas se tendrán al final. Simulaciones para $S_{\alpha}=10^{-9}$ mostraron que, mismo el factor de generación siendo tan pequeño, el comportamiento del flujo bifásico se queda igual, o sea, las burbujas aún son arrastradas por el fluido y se acumulan en las mismas regiones, paredes superiores y zonas con menor velocidad.

Por otro lado, con las burbujas de diámetro nanometrico, el comportamiento del flujo bifásico fue más estable y el factor de generación no há presentado gran influencia. Para este diámetro, resultados con las mallas M1 y M2 ya fueron satisfactorios. Se puede observar en la Ilustración 6 que para la malla M2 y un valor de generación de $10^{-4}$, cuando el Re es igual a 500 las burbujas de nano diámetro han afectado poco la hidrodinamica del fluido, mientras que si reducido el $\operatorname{Re}$ a 10 , la hidrodinámica pasa a estar influenciada por la presencia de las burbujas. A pesar de esto, esta influencia aun es mucho menor que con el tamaño micro. De hecho, la velocidad máxima es 2 ordenes de magnitud menor para el tamaño nano (Ilustración 4).

Además, es posible observar también que para diferentes velocidades, las burbujas se concentran en lugares diferentes. Para la velocidad baja, en el canal inferior de entrada se forma un vórtice grande antes de la curva U. Debido a este vórtice, las burbujas se acumulan en la pared superior del canal de entrada. Después de pasar por la curva, las burbujas se acumulan en la pared superior debido a otro gran vórtice que se forma en este canal. En contrapartida, para el Reynolds alto, la velocidad de entrada del líquido es más alta y tiene más fuerza para provocar una fluidización 
más estable. Por tanto, en este caso, lo que ocurre es que las burbujas son arrastradas por el metal líquido y se acumulan más en el canal superior en las paredes donde la velocidad del líquido es casi nula (Ilustración 7).

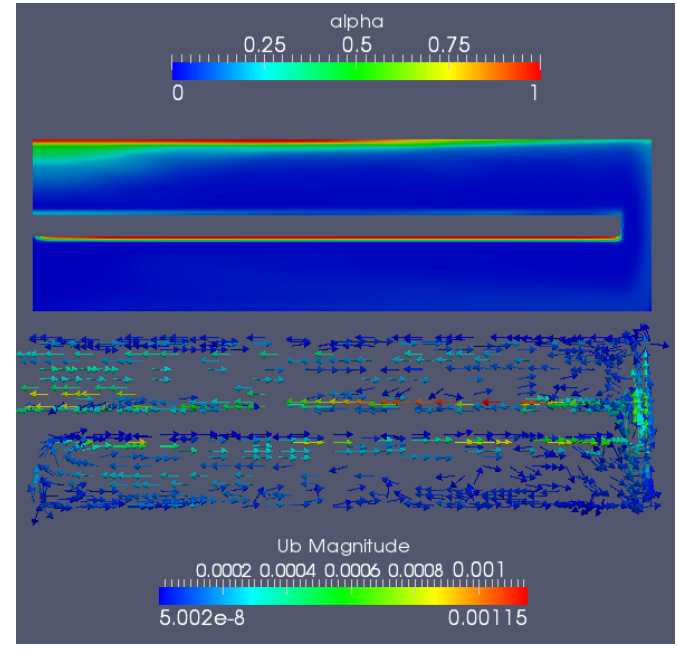

(a)

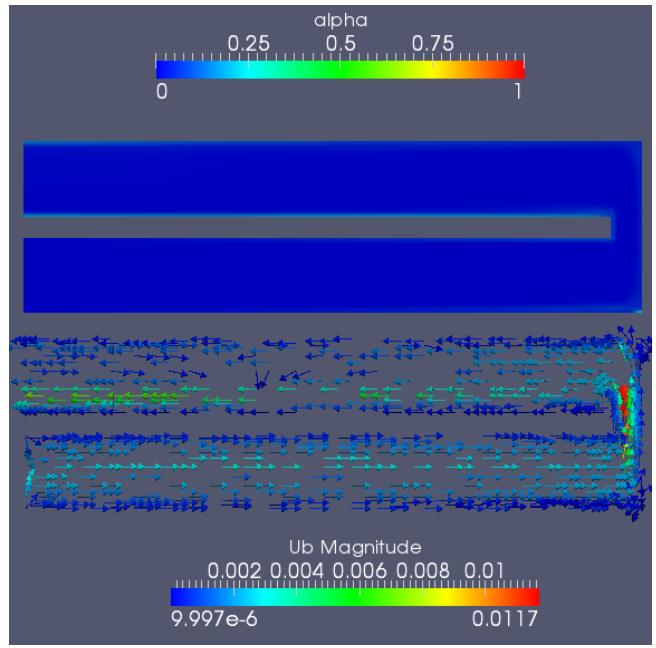

(b)

Ilustración 6 - Soluciones para nano burbujas con $S_{\alpha}=10^{-4}$. (a) $\operatorname{Re}=10$ y (b) $\operatorname{Re}=500$. (M2)

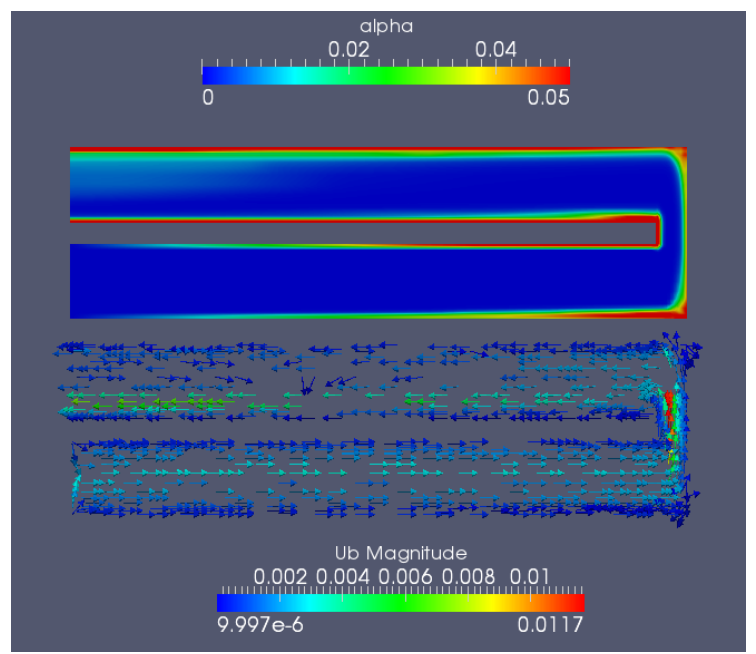

Ilustración 7 - Solución para nano burbujas, $\mathrm{S}_{\alpha}=10^{-4}$ y $\mathrm{Re}=500$ con leyenda mejorada (M2).

\section{CONCLUSIONES}

Las burbujas de helio formadas en el canal del breeding blanket del tipo HCLL pueden afectar considerablemente la hidrodinámica del metal líquido, lo que confirma la importancia de este estudio. En las simulaciones obtenidas, se ha observado la acumulación de burbujas en las paredes superiores y zonas de velocidad baja. Esto puede afectar la transferencia de masa y calor dentro de los canales de 
metal líquido, lo que puede alterar la permeación del tritio y dar lugar a una baja eficiencia térmica de los breeding blankets.

A modo de resumen, sobre el comportamiento físico de los resultados obtenidos es posible afirmar que el tamaño de la burbuja es el parámetro más importante y que las micrométricas han presentado gran influencia en la hidrodinámica del líquido, mientras las nanométricas la han afectado muy poco. El parámetro del factor de generación prácticamente no han presentado un impacto considerable. Y el Reynolds, para micro burbujas, tampoco, mientras que para las nano burbujas, que son más ligeras, el Reynolds más elevado ha disminuido el impacto de las burbujas en la hidrodinámica.

\section{NOMENCLATURA}

$\begin{array}{lll}\alpha & \text { Fracción de volumen de la fase dispersa } & {[-]} \\ \rho & \text { Densidad } & {\left[\mathrm{kg} / \mathrm{m}^{3}\right]} \\ \varphi & \text { Fase dispersa o contínua } & {[-]} \\ \mathrm{d} & \text { Diámetro de las partículas } & {[\mathrm{m}]} \\ \mathrm{g} & \text { Aceleración de gravedad } & {\left[\mathrm{m} / \mathrm{s}^{2}\right]} \\ \mathrm{M} & \text { Transferencia de momento en la interface } & {\left[\mathrm{kg} \cdot \mathrm{m}^{2} / \mathrm{s}^{2}\right]} \\ \mathrm{p} & \text { Presión } & {[\mathrm{Pa}]} \\ \mathrm{Re}_{\text {eff }} & \text { Tensor de tensiones de Reynolds } & {[-]} \\ \mathrm{S} & \text { Término de generación de burbujas de helio } & {\left[\mathrm{N} / \mathrm{m}^{2}\right]} \\ \mathrm{t} & \text { Tiempo } & {[\mathrm{s}]} \\ \mathrm{U} & \text { Vector de velocidad } & {[\mathrm{m} / \mathrm{s}]}\end{array}$

\section{REFERENCIAS}

BATET, L.; FRADERA, J.; MAS DE LES VALLS, E.; SEDANO, L. A. Numeric implementation of a nucleation, growth and transport modelo for helium bubbles in lead-lithium HCLL breeding blanket channels: Theory and code development. Fusion Engineering and Design, v. 86, p. 421-428, 2011

CONRAD, R.; DEBARBERIS, L.; COEN, V.; FLAMENT, T. Irradiation of liquid breeder material $\mathrm{Pb}-17 \mathrm{Li}$ with in-situ tritium release measurements in the LIBRETTO 2 experiment, Journal of Nuclear Materials, v. 179-181, part 2, p. 875-878, 1991

MAS DE LES VALLS, E.; SEDANO, L. A.; BATET, L.; RICAPITO, I.; AIELLO, A.; GASTALDI, O.; GABRIEL, F. Lead-lithium eutectic material database for nuclear fusion technology. Journal of Nuclear Materials, v. 376, p. 353-357, 2008.

PERRY, R. H.; BENSKOW, L. R.; BEIMESCH, W. E. Perry's Chemical Engineers' Handbook. $8^{\text {th }}$ ed., New York, NY: McGraw-Hill, 2008. 